\title{
The effect of head tilt on meridional differences in acuity: Implications for orientation constancy*†
}

\author{
MARTIN S. BANKS†† \\ Center for Research in Human Learning, Institute of Child Development, University of Minnesota \\ Minneapolis, Minnesota 55455
}

and

\author{
STEPHANIE J. STOLARZ \\ University of Califormia, San Diego, La Jolla, Califormia 92037
}

\begin{abstract}
Horn and Hill (1969) and others have reported that a small number of units in the cat visual cortex undergo changes in receptive field orientation associated with body tilt. Such units reportedly compensate for tilt and may represent a mechanism for human orientation constancy. To test this, we measured meridional differences in visual acuity for head-vertical and head-tilted viewing conditions. The results of Experiment 1 did not directly support or refute the involvement of tilt-compensatory units. The results of Experiment 2, in which we controlled for countertorsion of the eyes, showed that meridional acuity differences correspond to the retinal and not the spatial orientation of the stimulus. We conclude that tilt-compensatory cortical units are not involved in human orientation constancy. The physiological evidence indicating the existence of tilt-compensatory units in the visual cortex is also reexamined.
\end{abstract}

If an observer tilts his head laterally while viewing a stationary object, he is well aware that the object remains stationary despite the resulting rotation of the object's image on his retina. This phenomenon of visual stability during head or body tilting is called orientation constancy. It implies a comparison at some level of the nervous system between the rotation of the retinal image and movement of the observer himself. Two types of cues indicating magnitude and direction of tilt could provide the orientation information necessary for this comparison: vestibular cues arising from the labyrinths and kinaesthetic cues arising from the muscular and arthrodial (joint) afferent systems. Relevant in this regard are studies of the effects of nonvisual input on single-unit activity in the visual cortex. Specific and nonspecific vestibular afferents have been shown to modify the activity of single units in the mammalian visual cortex (Jung,

\footnotetext{
*We wish to acknowledge the assistance of Professors Robert Livingston, David Rumelhart, and Harry Munsinger of the University of California, San Diego, in obtaining some of the equipment used in this study. We also thank Professors Philip Salapatek and Dwight Burkhardt of the University of Minnesota for comments on an earlier draft.

tWe have recently learned that Lennie (1974) has performed an experiment similar to our Experiment 1. Also, Wade (1973) has sought evidence for tilt-compensatory cortical units by studying the face of linear afterimages. Both authors failed to find evidence for tilt-compensatory units.

††Reprint requests should be sent to: Martin S. Banks, Center for Research in Human Learning, 205 Elliott Hall, University of Minnesota, Minneapolis, Minnesota 55455.
}

1961; Jung, Kornhuber, \& DaFonseca, 1963). Also, somatic stimulation activates many units within the visual cortex (Horn, 1965; Murata, Cramer, \& Bach-y-Rita, 1965).

Evidence that vestibular or kinaesthetic afferent information may be directly involved in orientation constancy comes from studies of the effect of body tilt on the responsivity of cortical units in animals. Under untilted viewing conditions, mammalian cortical units are typically found to respond selectively to bars or edges of a particular orientation (Hubel \& Wiesel, $1962,1968)$. Recent studies of the cat visual cortex have shown that when the animal's head or body is tilted laterally, a small percentage of cortical units undergo a change in receptive field orientation such that the optimal stimulus orientation is unaltered with respect to gravitational vertical (Horn \& Hill, 1969; Horn, Stechler, \& Hill, 1972; Spinelli, 1970; Denney \& Adorjani, 1972). Thus, these units vary in responsivity according to the spatial and not the retinal orientation of the stimulus. Similar properties for units in the cat superior colliculus have been reported (Bisti, Maffei, \& Piccolino, 1972). These units exhibit the property of tilt compensation or orientation constancy themselves and, therefore, may represent a mechanism for the perceptual phenomenon of orientation constancy.

Recently, attempts have been made to find a human psychophysical correlate of tilt-compensatory units. Findlay and Parker (1972) and Mitchell and Blakemore (1972) employed the threshold-elevation 
aftereffect under various conditions of head tilt and stimulus orientation. They found that threshold elevation is greatest when the adapting and test stimuli are of similar retinal, rather than spatial, orientation. They concluded that orientation constancy does not exist at the neural site of adaptation in the threshold-elevation aftereffect. The site has been shown to be in the human visual cortex or higher (Blakemore \& Campbell, 1969). It is possible, however, if tilt-compensatory units are involved to a limited extent at the neural site of the aftereffect, that threshold elevation could still correspond more closely to retinal than to spatial stimulus orientation. In fact, as Horn and Hill (1969), Horn et al (1972), and Denney and Adorjani (1972) have pointed out, only a limited set of cortical units exhibit properties of orientation constancy, indicating the improbability of a shift in the orientation selectivity of the entire visual system when the head is tilted from a vertical to an oblique position. Thus, to test the involvement of a limited set of tilt-compensatory units, experimental measurements should be accurate enough to determine whether perceptual performance corresponds exactly to retinal stimulus orientation or not. Since figural aftereffects are subject to a high degree of perceptual variability, a simple detection paradigm might be more appropriate than the adaptation paradigm used by Findlay and Parker (1972) and Mitchell and Blakemore (1972).

Detection of checkerboards or gratings presented at various orientations has been used to investigate orientation constancy. With untilted viewing, visual acuity is generally found to be greatest for vertical or horizontal lines and gratings and poorest for oblique lines and gratings (reviewed by Taylor, 1963; Howard \& Templeton, 1966). This acuity asymmetry, called visual anisotropy, exists in the absence of clinical astigmatism, and cannot be accounted for by meridional differences in the retinal mosaic (Polyak, 1957), directional preference for eye movements (Nachmias, 1960), or dioptric meridional asymmetry (Campbell, Kulikowski, \& Levinson, 1966; Mitchell, Freeman, \& Westheimer, 1967). Thus, visual anisotropy appears to be the result of neural processing differences dependent on stimulus orientation. If these processing differences could be shown to correspond to the spatial and not the retinal orientation of the stimulus, this would support the hypothesis that orientation constancy in humans is the result of tilt compensation at the level of the visual cortex.

Luria (1963) measured the detectability of checkerboard patterns of variable fineness presented scotopically at vertical and oblique orientations under various conditions of head and body tilt. He found acuity at 45-deg head or body tilt to be greatest for a checkerboard presented obliquely $(45 \mathrm{deg}$ from gravitational vertical), indicating that meridional variations in acuity depend on stimulus orientation on the retina rather than orientation with respect to gravity. ${ }^{1}$ His data also show, however, that meridional differences are less for tilted ( $45 \mathrm{deg}$ ) viewing than for vertical. Therefore, it is inappropriate to directly conclude from Luria's results that tilt-compensatory mechanisms are not involved, since the magnitude of anisotropy is shown to vary as a function of head or body tilt.

Findlay and Parker (1972) also measured meridional acuity as a function of head tilt. The stimuli used were high-frequency sine-wave gratings ( 24 cycles/deg) presented at a photopic level of illumination. Their results are similar to Luria's in that, when the observer's head was tilted $45 \mathrm{deg}$, maximum acuity was obtained for oblique stimuli. Their results are dissimilar, however, in that meridional differences were found to be greater with 45-deg head tilt than with vertical viewing. The authors concluded that there was no evidence for tilt compensation at the level of grating detection.

This paper reports measurements taken under conditions similar to those of Findlay and Parker. However, the meridional differences as a function of head tilt are shown to be consistent with Luria's findings and not theirs. This ambiguous result is resolved in a second experiment in which differences between retinal orientation and head orientation due to countertorsion of the eyes are considered.

\section{METHOD}

\section{Apparatus}

Sine-wave gratings were generated on a Tektronix 560 oscilloscope in a manner similar to that described by Campbell and Green (1965). They were displayed on a Tektronix 602 display scope such that the space-average luminance was constant $(0.5 \mathrm{fL})$ and the gratings were continuously variable in contrast and spatial frequency. ${ }^{2}$ The observers viewed the stimuli monocularly with a natural pupil through a specially designed optical tunnel such that only the stimulus field and field surround could be seen. The stimulus field was circular and subtended $5 \frac{1}{2} \mathrm{deg}$. The field surround subtended $25 \mathrm{deg}$, was unpatterned, and matched the stimulus field in both average luminance and hue. Observers held a five-turn logarithmic potentiometer which they used to vary the Z-axis modaulting voltage of the display scope and hence the contrast of the gratings. A 20-dB-step attenuator could also be adjusted by the experimenter. The orientation of the gratings was manipulated by tilting the portable display scope to the desired values. Head tilt was accomplished by an adjustable device which could be tilted to 45 or $51 \mathrm{deg}$ from vertical. Attached to this device were a cupped chinrest and head clamp (both fitted to the individual subjects), which were fastened together to hold the tilt of the observer's head constant. Observers sat in an upright chair so that tilting of the trunk was insignificant.

\section{Procedure}

The emmetropic authors, who were considerably practiced at the experimental task, served as observers (M.S.B. in Experiment 1 and M.S.B. and S.J.S. in Experiment 2). Observers were dark-adapted for $5 \mathrm{~min}$ before the beginning of an experimental session. Head tilt, stimulus orientation, and spatial frequency were set to the appropriate values for the condition to be run. The observer then adjusted the contrast of the grating until it was judged to be at the threshold of detectability. ${ }^{3}$ The $Z$-axis modulating voltage was recorded by the experimenter for that 


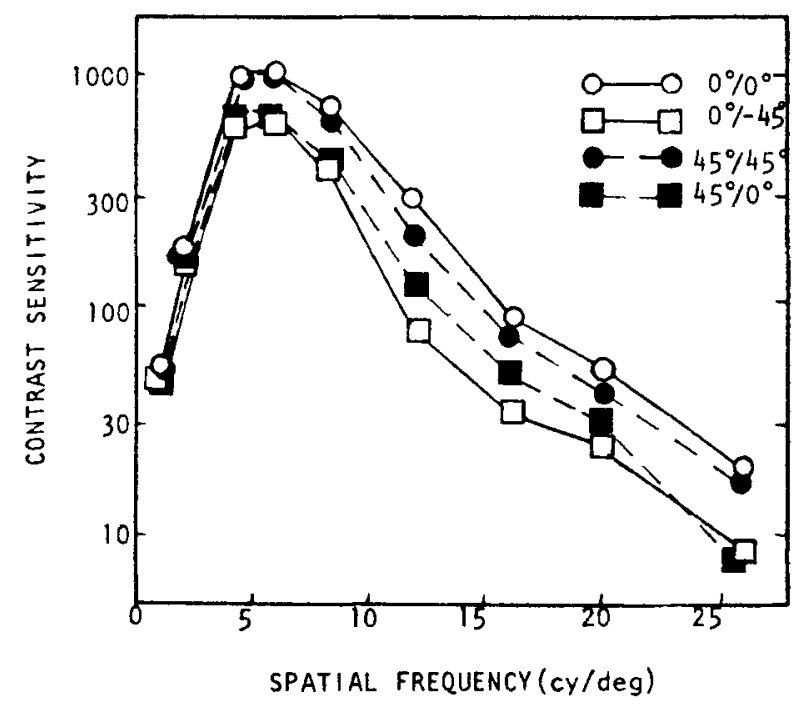

Fig. 1. Contrast sensitivity as a function of spatial frequency for four head-tilt/grating-orientation conditions. Open circles and solid lines represent the contrast sensitivity function for 0 -deg (vertical) head tilt and 0 -deg (vertical) grating orientation; open squares and solid lines, the function for 0 -deg head tilt and $45-\mathrm{deg}$ (oblique) grating orientation; filled circles and broken lines, the function for 45-deg head tilt and 45-deg grating orientation; and filled squares and broken lines, the function for 45 -deg head tilt and 0-deg grating orientation. Observer M.S.B.

setting. The potentiometer was then randomly spun and the observer adjusted the grating contrast to threshold once more. In Experiment 1, three observations were obtained before stimulus condition was changed; in Experiment 2, six or seven were obtained. The next stimulus condition to be run was chosen randomly.

\section{RESULTS}

\section{Experiment 1}

Contrast sensitivity measurements were obtained for a number of spatial frequencies at the following head-tilt/grating-orientation conditions: ${ }^{4} 0 \mathrm{deg} /$ $0 \mathrm{deg}$, (2) $0 \mathrm{deg} /-45 \mathrm{deg}$, (3) $45 \mathrm{deg} / 45 \mathrm{deg}$, and (4) $45 \mathrm{deg} / 0 \mathrm{deg}$. Figure 1 shows the contrast sensitivity functions obtained. Each plotted point represents the mean of six observations. The ordinate is contrast sensitivity ( 1 /contrast at threshold) with contrast defined by: $\mathrm{C}=\left(\mathrm{L}_{\max }-\mathrm{L}_{\min }\right) /\left(\mathrm{L}_{\max }+\right.$ $\mathrm{L}_{\min }$ ), where $\mathrm{L}_{\max }$ refers to the luminance of the brightest portion of the stimulus and $\mathrm{L}_{\min }$ to the dimmest. The classical anisotropic effect is exemplified by the decreased high-frequency sensitivity of the $0 /-45-\mathrm{deg}$ function in comparison to the $0 / 0$-deg function. The magnitude and frequency range of the sensitivity asymmetry agree with other's measurements (Campbell et al, 1966; Mitchell et al, 1967). More noteworthy is the sensitivity difference between the 45/45-deg and 45/0-deg functions, the former showing greater high-frequency contrast sensitivity. One might conclude from this that meridional acuity differences correspond to stimulus orientation on the retina. However, 0/-45-deg sensitivity is less than $45 / 0 \mathrm{deg}$. Similarly, $45 / 45-\mathrm{deg}$ sensitivity is less than $0 / 0 \mathrm{deg}$. Thus, there is the suggestion that contrast sensitivity does not exactly correspond to the retinal stimulus orientation in that the meridional difference is less for the head-tilted than for the head-vertical functions.

To better illustrate this, Fig. 2A displays the results for one spatial frequency ( 20 cycles $/ \mathrm{deg}$ ). Contrast sensitivity is plotted as a function of stimulus orientation. ${ }^{5}$ Separate functions are shown for the 0 and 45-deg head-tilt conditions. All-or-none interpretations of two hypotheses relating anisotropy to head tilt are also displayed. If meridional differences in contrast sensitivity corresponded to the spatial orientation of the stimulus, as might be suggested by the physiological findings of Horn and Hill and others, then the 45-deg head-tilt function shown as a dotted line would be obtained. Maximum detectability would occur at a stimulus orientation of $0 \mathrm{deg}$ whether the head was tilted or not. If, on the other hand, meridional differences in contrast sensitivity followed retinal orientation, then maximum detectability would coincide with the degree of head tilt. This hypothesis, which disregards countertorsion of the eys, is shown as a dashed line. It is clear from this figure that our data as presented do not correspond to either hypothesis. The anisotropic effect for the head-tilted viewing condition is smaller than for the head-vertical condition.

Findlay and Parker's data are plotted in a similar manner in Fig. 2B. Here, however, $d^{\prime}$, rather than contrast sensitivity, is represented on the ordinate since their response measure was observer sensitivity for constant contrast gratings as a function of head tilt and grating orientation. The results for two observers have been averaged. In this case, a correspondence between the simple retinal hypothesis and the data is evident, but the anisotropic effect is larger for head-tilted viewing than it is for head-vertical viewing.

Luria's scotopic data, when displayed in a similar manner, appear to concur with ours. Figure $2 \mathrm{C}$ plots fineness of just-detectable checks in a checkerboard as a function of head tilt and stimulus orientation. The results for four observers have been averaged. It is evident that neither the simple retinal nor the spatial hypothesis is directly supported by these results. The decreased anisotropic effect in the head-tilted condition of Luria's and our results could be interpreted as evidence for a partial tilt compensation; that is, something of a compromise between the retinal and spatial hypotheses.

\section{Experiment 2}

A fact which comes to bear in resolving the ambiguity of Luria's and our findings is the countertorsion of the eyes when the head is tilted (reviewed by Howard \& Templeton, 1966). It is 


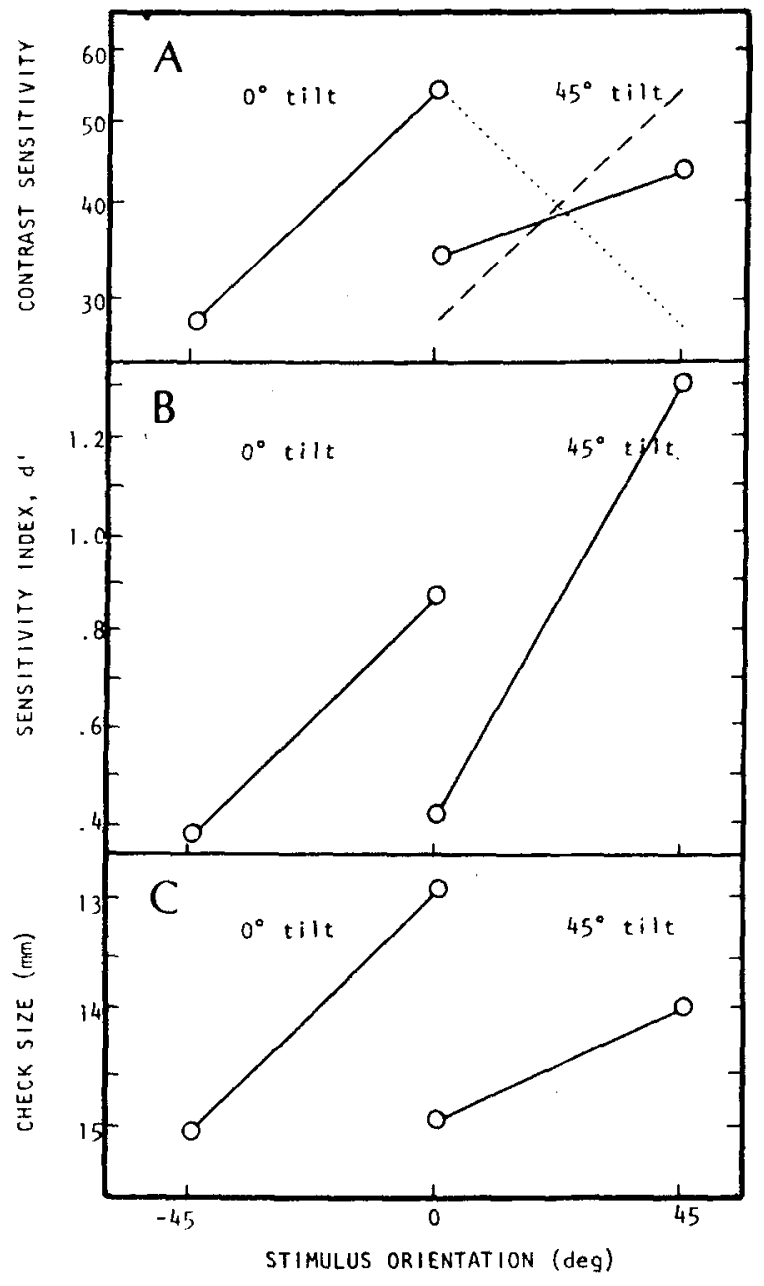

Fig. 2. Acuity as a function of stimulus orientation (re: gravitational vertical) for head-vertical $(0 \mathrm{deg})$ and head-tilted (45 deg) viewing conditions. (A) Contrast sensitivity vs stimulus orientation from Experiment 1. The open circles and solid lines represent experimentally obtained data for 20 -cycle/deg sine-wave gratings. Two viewing conditions, head-vertical and head-tilted, are represented. Observer M.S.B. The dotted and dashed lines represent theoretical acuity functions for the head-tilted viewing condition. If meridional differences in acuity correspond to the spatial orientation of the stimulus, the dotted line should be obtained. If meridional differences correspond to the retinal orientation of the stimulus, the dashed line should be obtained. (B) Observer sensitivity (d') vs stimulus orientation from Findlay and Parker (1972), Two viewing conditions, head-vertical and head-tilted, for 24-cycle/deg gratings. Data are averaged from two observers. (C) Just-detectable check size vs stimulus orientation from Luria (1963). Two viewing conditions, head-vertical and head-tilted, for checkerboard patterns. Data averaged from four observers.

commonly reported that, when the head is tilted laterally, both eyes rotate slightly in the opposite direction. Consequently, orientation of the retina does not correspond to orientation of the head when it is tilted. In the three sets of data displayed in Fig. 2, however, it has been implicitly assumed that orientation of the retina is equal to head orientation, ${ }^{6}$
To rectify this error, a second experiment was conducted in which retinal stimulus orientation was more accurately specified. A head-tilt value of $51 \mathrm{deg}$ was chosen because at this head orientation the eyes counterroll $6 \mathrm{deg}$, resulting in a retinal orientation of $45 \mathrm{deg}$ (Schoene, 1962; J. R. Nelson, personal communication, 1973). This procedure thus allows a more appropriate comparison of meridional differences in the head-vertical and head-tilted viewing conditions.

Contrast sensitivity was measured for a high-frequency sine-wave grating (20 cycles/deg) under the following head-tilt/grating-orientation conditions: $0 / 0,0 /-22.5,0 / 45,51 / 0,51 / 22.5$, and $51 / 45 \mathrm{deg}$. Twenty observations (six or seven at a time) were obtained for each stimulus condition. Figure 3 shows the results for two observers. Each plotted point represents the mean of 20 observations. ${ }^{7}$ The classical anisotropic effect is shown by differences in sensitivity for the $0 / 0-, 0 /-22.5-$, and $0 /-45-\mathrm{deg}$ viewing conditions, Most important, however, is the close parallelism between the head-vertical and head-tilted conditions for both observers. The retinal stimulus orientation is equivalent for the $0 /-45$ - and $51 / 0-$ deg conditions, the $0 /-22.5-$ and $51 / 22.5-\mathrm{deg}$ conditions, and the $0 / 0-$ and $51 / 45-\mathrm{deg}$ conditions. Figure 3 shows that contrast sensitivity was approximately equal for each of these three pairs. This finding strongly supports the conclusion that meridional differences in acuity correspond to retinal rather than spatial orientation.

\section{DISCUSSION}

There is no apparent explanation for the discrepancy between Findlay and Parker's measurements and those of Experiment 1. The use of observer sensitivity (d') for constant contrast gratings rather

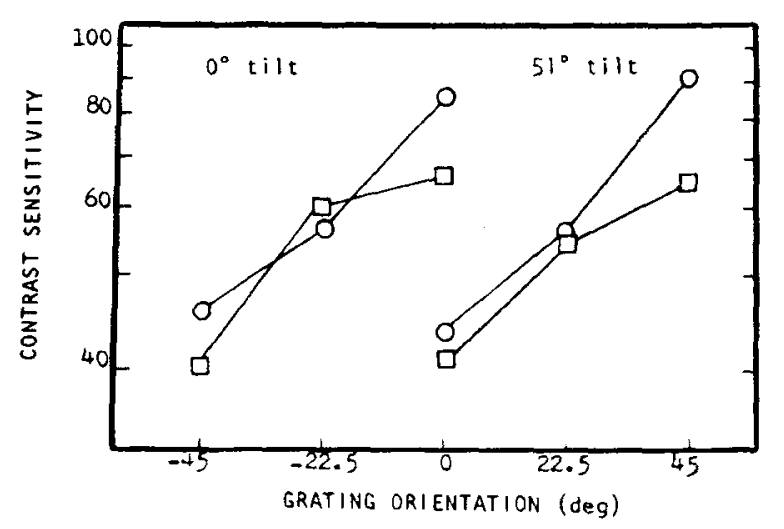

Fig. 3. Acuity as a function of stimulus orientation (re: gravitational vertical) from Experiment 2. Contrast sensitivity is plotted for 20-cycle/deg gratings viewed with head vertical (0 deg) and head tilted (5I deg). Open circles and solid lines represent data from Observer M.S.B. Open squares and solid lines represent data from Observer S.J.S. 
than contrast at threshold should not have led to the qualitative difference in results.

Luria discounted the effect of eye countertorsion in explaining the difference in the magnitude of anisotropy between head-tilted and head-vertical viewing conditions. The results of Experiment 2, however, show that if one controls for countertorsion, this difference is eliminated.

The striking correspondence between contrast sensitivity and retinal stimulus orientation does not support the involvement of tilt-compensatory units at the level of grating detection. The question should then be raised, to what extent does this result actually refute the hypothesis that such units are involved at that level? The present data, when considered in conjunction with recent psychophysical and physiological experimentation, do, in fact, suggest that tilt-compensatory units are not involved to any significant degree in cortical processing associated with grating detection.

Recent psychophysical research (Gilinsky, 1968; Blakemore \& Campbell, 1969) suggests that the feature-extracting properties of the human visual cortex are fundamentally similar to those studied electrophysiologically in cats and monkeys (Hubel \& Wiesel, 1962, 1968; Campbell, Cleland, Cooper, \& Enroth-Cugell, 1968; Maffei, Fiorentini, \& Bisti, 1973). The tilt-compensatory units originally reported by Horn and Hill are cortical and thus exhibit the same size and orientation selectivity expected of higher mammalian cortical units. One would therefore expect to find human psychophysical correlates of their functional properties by using the paradigms employed to demonstrate size and orientation selectivity in the human visual system. A paradigm using human visual anisotropy is appropriate for the following reasons. Electrophysiological study of the cat visual cortex finds a somewhat greater number of units responsive to vertical or horizontal stimuli than to oblique stimuli (Henry, Dreher, \& Bishop, 1974). This preference for vertical and horizontal orientations provides a feasible mechanism for anisotropy. Extrapolating from these cat data to the human visual system, one might reasonably assume that the set of human cortical units responsive to vertically or horizontally oriented stimuli has a greater number of units responsive to small bar widths or high spatial frequencies in comparison to the set of units responsive to oblique stimuli. This notion is supported by studies of the visually evoked cortical potential in humans (Campbell \& Maffei, 1970; Maffei \& Campbell, 1970). Now consider the 51-deg head tilt condition of our Experiment 2. If a significant number of the units normally sensitive to vertical stimuli underwent receptive field orientation change to compensate for tilting of the retina, sensitivity to obliquely oriented, high-frequency gratings should increase. Thus, to the extent that an assumption of convergence between the functional properties of cortical units in higher mammals and the mechanism of visual anisotropy in humans is valid, the negative results of this report imply that tilt-compensatory units are not significantly evident in the human visual cortex. The negative results of studies using the threshold-elevation aftereffect lend greater weight to this conclusion.

Given the inability to find psychophysical correlates of tilt-compensatory units, a brief look at more recent physiological evidence is appropriate. Schwartzkroin (1972) has reported a failure to replicate the finding of Horn and Hill and others. He studied the orientation selectivity of 33 cortical units in the cat and found that three exhibited tilt-compensatory properties. The receptive-field orientation changes of these three units, however, were shown to correspond to spontaneous countertorsion of the eyes. ${ }^{8}$ Schwartzkroin has also presented two important criticisms of the interpretations of Horn and Hill and others. First, large spontaneous changes in the receptive fields of cortical units occur in the absence of body tilt. Such alterations in size and shape, not specifically correlated with body tilting, could lead to an incorrect interpretation of orientation selectivity drift under body tilt. Second, it has been shown that nonspecific arousal can cause changes in the receptive fields of visual units (Meulders \& Godfraind, 1969). Body tilting could be one of many potentially arousing stimuli. It should also be noted that Denney and Adorjani's results exhibit a negligible correlation between the magnitude and direction of head tilt and the magnitude and direction of change in receptive field orientation. This is, of course, inconsistent with properties required of a tilt-compensatory mechanism. Horn et al (1972) reported that 14 of 224 units studied exhibited receptive field orientation change with body tilt. Thirteen of these units showed changes opposite to the direction of tilt (which was interpreted as tilt compensation). The authors, however, report the magnitude of change for only three "tilt-compensating" units (their Figs. 5, 7, and 8) and, for two of these units, the magnitude of change is not correlated with the magnitude of tilt. Thus, considering the small proportion of units which exhibit receptive field orientation change and the lack of correlation between that change and the degree of body tilt, it seems unlikely, from the physiological as well as the psychophysical evidence, that tiltcompensatory cortical units, as originally proposed by Horn and Hill, are significantly involved in orientation constancy.

\section{REFERENCES}

Bisti, S., Maffei, L., \& Piccolino, M. Variations of the visual responses of the superior colliculus in relation to body roll. Science, 1972, 175, 456-457.

Blakemore, C., \& Campbell, F. W. On the existence of neurones in the human visual system selectively sensitive to the orientation and size of retinal images. Journal of Physiology, 1969, 203, 237-260.

Campbell, F. W., Cleland, B. G., Cooper, G. F., \& 
EnRoth-Cugell, C. The angular selectivity of visual cortical cells to moving gratings. Journal of Physiology, 1968, 198, 237-250.

Campbell, F. W., \& Green, D. G. Optical and retinal factors affecting visual resolution. Journal of Physiology, 1965, 181, 576-593.

Campbell, F. W., Kulikowski, J. J., \& Levinson, J. The effect of orientation on the visual resolution of gratings. Journal of Physiology, 1966, 187, 427-436.

Campbell, F. W., \& MAFFei, L. Electrophysiological evidence for the existence of orientation and size detectors in the human visual system. Journal of Physiology, 1970, 207, 635-652.

DENNEY, D., \& ADoRJANI, C. Orientation specificity of visual cortical neurons after head tilt. Experimental Brain Research, 1972, 14, 312-317.

FindLAY, J. M., \& PARKer, D. M. An investigation of visual orientation constancy using orientation-specific properties of acuity and adaptation. Perception, 1972. 1, 305-313.

GILINSKY, A. S. Orientation-specitic effects of patterns of adapting iight on visual acuity. Journal of the Optical Society of America, 1968, 58, 13-18.

Henry, G. H., Dreher, B., \& Bishop, P. O. Otientation specificity of cells in the cat striate cortex. Journal of Neurophysiology, 1974, in press.

HorN, G. The effect of somaesthetic and photic stimuli on the activity of units in the striate cortex of unanesthetised. unrestrained cats. Journal of Physiology, 1965, 179, 263-277.

Horn, G., \& Hill, R. M. Modifications of receptive fields in cells in the visual cortex occurring spontaneously and associated with bodily tilt. Nature (London), 1969, 221, 186-188.

Horn, G., Stechler, G., \& Hill, R. M. Receptive fields of units in the visual cortex of the cat in the presence and absence of bodily tilt. Experimental Brain Research, 1972, 15, 113-132.

Howard, I. P, \& Templeton, W. B. Human spatial orientation. London: Wiley, 1966.

Hubel, D. H., \& WiESEL, T. N. Receptive fields, binocular interaction and functional architecture in the cat's visual cortex. Journal of Physiology, 1962, 160, 106-154.

Hubel, D. H., \& Wiesel, T. N. Receptive fields and functional architecture of monkey striate cortex. Journal of Physiology, 1968, 195, 215-243.

JunG, R. Neuronal integration in the visual cortex and its significance for visual information. In W. Rosenblith (Ed.), Sensory communication. New York: Wiley, 1961. Pp. 627-674.

Jung, R., Kornhuber, H. H., \& DaFonseca, J. S. Multisensory convergence on cortical neurons. Progress in Brain Research, 1963, 1, 207-234.

Kelcy, D. H. Bars, checks and color effects. In Abstracts of the Association for Research in Vision and Ophthalmology Meeting. Sarasota, Florida, 1974, p. 101.

LENNIE, P. Head orientation and meridional variations in acuity. Vision Research, 1974, 14, 107-111.

LuRIA, S. M. The effect of body position on meridional variations in scotopic acuity. American Journal of Psychology, 1963, 76, 598-606.

Maffei, L., Fiorentini, A., \& Bisti, S. Neural correlate of perceptual adaptation to gratings. Science, 1973, 182, $1036 \cdot 1038$.

MAFFeI, L., \& CAMPBell, F. W. Neurophysiological localization of the vertical and horizontal visual coordinates in man. Science, 1970, 167, 386-387.

Meulders, M.. \& Godfraind, J. M. Influence du réveil d'origine réticulaire sur l'étendue des champs visuels des neurones de la région genouillée chez le chat avec curveau intact ou avec cervean isolé. Experimental Brain Research, $1969,9,201-220$.

Mitchell. D. E., \& Blakemore, C. The site of orientation constancy. Perception, 1972, 1, 315-320.

Mitchell, D. E., Freeman, R. D., \& Westheimer, G. Effect of orientation on the modulation sensitivity for interference fringes on the retina. Joumal of the Optical Society of America. $1967,57,246-249$.
Murata, K., Cramer, H., \& Bach-Y-Rita, P. Neuronal convergence of noxious, acoustic and visual stimuli in the visual cortex of the cat. Journal of Neurophysiology, 1965, 28, 1223-1238.

NaChmias, J. Meridional variations in visual acuity and eyemovement during fixation. Journal of the Optical Society of America, 1960, 50, 569-571.

Polyak, S. The vertebrate visual system. Chicago: University of Chicago Press, 1957.

Schoene, H. Ueber den Einfluss der Schwerkraft auf die Augenrollung und auf die Wahrnehmung der Lage im Raum. Zeitschrift fur Vergleichende Physiologie, 1962, 46, 57-87.

Schwartzkroin, P. A. The effect of body tilt on the directionality of units in cat visual cortex. Experimental Neurology, 1972, 36, 498-506.

SPINELLI, D. N. Recognition of visual patterns. In D. A. Hamburg (Ed.), Perception and its disorders. Baltimore: Williams and Watkins, 1970. Pp. 139-149.

TAYLOR, M. M. Visual discrimination and orientation. Journal of the Optical Society of America, 1963, 53, 763-765.

$W_{A D E}$, N. J. Orientation and spatial frequency effects on linear afterimages: The retinal reference for selectivity-a supplementary report. Perception \& Psychophysics, 1973, 14, 384-386.

\section{NOTES}

1. Kelly (1974) has shown that the fundamental Fourier components of checkerboard patterns are 45-deg diagonals. From this analysis, one would predict greater visibility for obliquely oriented than for vertically oriented checkerboards, which is the reverse of Luria's results.

2. Space-average luminance and grating contrast were measured photometrically with a Spectra brightness spot meter. Space-average luminance was uniform across the stimulus field. Using a 7 -cycle/deg square-wave grating, we measured contrast as a function of input voltage. A linear relationship obtained for a contrast range of 0 to 0.4 . Contrast at a fixed input voltage was also measured over a spatial frequency range of 0.4 to $26 \mathrm{cycles} / \mathrm{deg}$ and was found to be constant.

3. The method of adjustment was used to minimize the number of observations necessary for each stimulus condition. The small variability of the results (Notes 5 and 7 ) justifies this procedure.

4. The first digit refers to degrees of head tilt from vertical. The second digit refers to grating orientation in degrees of departure from gravitational vertical. Positive numbers refer to clockwise departure from vertical.

5. The standard errors for the plotted data points are: 8.1 for $0 / 0 \mathrm{deg} ; 4.0$ for $0 / 45 \mathrm{deg} ; 11.8$ for $45 / 45 \mathrm{deg}$; and 6.4 for $45 / 0$ deg.

6. Luria actually considered countertorsion of the eyes as the cause of the meridional differences between his head-vertical and head-tiited conditions. However, he rejected it as a complete explanation.

7. The standard errors for the plotted data points are:

\begin{tabular}{lccc} 
& $0 / 0$ Deg & $0 /-22.5 \mathrm{Deg}$ & $0 /-45 \mathrm{Deg}$ \\
\cline { 2 - 4 } MSB & 15.4 & 4.3 & 6.1 \\
SJS & 15.0 & 8.8 & 9.5 \\
& $51 / 0 \mathrm{Deg}$ & $51 / 22.5 \mathrm{Deg}$ & $51 / 45 \mathrm{Deg}$ \\
\cline { 2 - 4 } MSB & 8.9 & 5.6 & 12.1 \\
SJS & 11.0 & 10.8 & 18.0 \\
\hline
\end{tabular}

8. Horn and Hill (1969), Horn et al (1972), and Denney and Adorjani (1972) controlled for this potential artifact by physically restraining torsion of the stimulated eye.

(Received for publication May 20, 1974; revision received July $30,1974$. 